Vorwort zur vierten Auflage.

In der vorliegenden neuen Auflage wurden die Präparate aus der aliphatischen Reihe um die Darstellung von Glykol aus Äthylenbromid vermehrt. Herrn Prof. Dr. L. Henky in Löwen bin ich für wertvolle Ratschläge bezgl. dieses Präparates zu großem Danke verpflichtet. Unter die aromatischen Präparate wurde ein Beispiel für die Reimer-Tiemannsche Synthese (Salicylaldehyd), sowie die Zerlegung der inaktiven Mandelsäure in ihre altiven Komponenten neu aufgenommen. - Die alten Vorschriften konnten beibehalten werden, wurden jedoch in manchen Punkten verbessert.

Zur Freude des Verfassers hat sein Buch auch im Auslande Beifall gefunden, wie das Erscheinen einer englischen Übersetzung beweist. Eine Übersetzung ins Russische befindet sich unter der Presse.

Heidelberg, im Februar 1900.

Gattermann

Vorwort zur funften Auflage.

Das vorliegende Buch ist ein unveränderter Abdruck der vierten Auflage. Mögen die Freunde des Büchleins demselben auch im alten Gewande treu bleiben.

Freiburg, im Oktober 1901.

Gattermann

\title{
Vorwort zur sechsten Auflage.
}

Dem Charakter des Buches entsprechend wurden in die neue Auflage zwei Beispiele für die so fruchtbare Grignardsche Reaktion neu aufgenommen. An den alten Vorschriften etwas zu ändern, erwies sich als nicht notwendig.

Freiburg, im Oktober 1903.

Gattermann

\section{Vorwort zur siebenten Auflage.}

Die neue Auflage unterscheidet sich von den früheren dadurch, daB theoretische Erörterungen, die für das organischpräparative Arbeiten von allgemeiner Bedeutung sind (Theorie der Wasserdampfdestillation, Teilungssatz, Massenwirkungsgesetz, Löslichkeitsprodukt), nach Möglichkeit Berücksichtigung fanden. Ferner wurde die Tabelle für die Berechnung der Stickstoffbestimmungen auf Grund des von RAYLEIGH und RAMSAX ermittelten Stickstoffgewichtes neu berechnet, bei welchem Anlasse auch die Mantissen der dekadischen Logarithmen eingefügt wurden.

Freiburg, im April 1905.

Gattermann 